\title{
Revealing the Phenomena of Heat and Photon Energy on Dealing Matter at Atomic Level
}

\section{Mubarak Ali}

Department of Physics, COMSATS Institute of Information Technology, 45550 Islamabad, PAKISTAN, mubarak74@comsats.edu.pk or mubarak74@mail.com, Ph.+92-51-90495406

Abstract -Technology is in the way to reach in its climax but the basic understanding of science in many phenomena is still awaited despite the fact that nature witnesses. Scientific research reveals strong analogy between electron and photon. Atoms of solid state behavior that execute suitable electron transitions excite electron at target while absorbing heat energy at shunt level. De-excitation of electron under the pulling force of nearby unfilled state available at bare surface of atom results into depicting force energy shape-like Gaussian distribution called unit photon where inertia involved at each stage of changing state. The continuous cycles of excitation and de-excitation of electron confined within inter-state (filled to unfilled and unfilled to filled) result into generate force energy in wave-like fashion propagating in the inter-state electron's gap of adjacent atoms in the lattice; in each unit photon, the force energy configures under electron's trajectory while excitation period is due to inertia-levitation-inertia behaviors and force energy configures under electron's trajectory while de-excitation period is due to inertia-gravitation-inertia behaviors. Silicon atom is considered as a model system of it. Uninterrupted confined inter-state electron-dynamics results into configure force energy that can travel immeasurable length where interruption from the point of generation termed it an overt photon -a long length photon. Such photons increase wavelength under decreasing energy when travelling in the medium other than interstate electron's gap where light glow is observed on attaining wavelength of their certain density in the visible range. They act as merged photons or squeezed photons while interacting (coordinating) to suitable medium, thus, on merging or squeezing convert into heat energy where atoms like silicon again configure them into force energy under the trajectory of electrons. Thus, heat energy dealing to suitable matter at atomic level 
transforms into photon energy. Involving levitation behavior in the course of exciting electron and gravitation behavior in the course of de-exciting electron validates that force of repulsion or attraction in certain materials engages the phenomenon of levitism or gravitism where inertia is exempted. Here, heat energy and photon energy explore matter at electron level. Thus, devise science to describe.

Keywords: heat energy; photon energy; materials science; nanoscale phenomenon; atomic scale phenomenon

\section{INTRODUCTION:}

Humanity is being benefited by heat energy and photon energy since the existence and electrical phenomena have been studied since long. Catching fire in various stuffs is a usual phenomenon known since the existence of life. Everyone is taking benefit of these blessings but the understanding behind these phenomena is not only clear and but also their coordination and interaction to various sorts of matter too.

A large number of studies are available in the literature dealing with light-matter interaction, and it has been covered largely under a phenomenon, namely, surface plasmons. Origin of plasmons was explored in some early published reports [1-4]. A plasmon is a quantum of plasma oscillation and represents the collective oscillations of the free electron gas density - a general definition extracted from the literature.

The interaction of light (photon) to matter is recognized in the form of various terminologies, namely, phonon, excitons, and plasmon, etc. Recently published review discusses the light-matter interaction by taking into account the properties of polaritons modes in two-dimensional materials and applications in the certain range of spectrum [5]. The concept of excitons (electron-hole pairs) was first proposed by Frenkel [6] and deals with excited state of an atom in a lattice travelling in particle-like fashion without net transfer of charge. Excitons can be formed on absorption of photon by a semiconductor (quantum dot) [7]; a phonon is a collective excitation in a periodic, elastic arrangement of atoms or molecules in condensed matter.

A vast number of studies deal the formation process of tiny particles -structure evolution at nanoscale. The tiny-sized cluster is a simple chemical compound which has variety of important applications in diversified areas [8]. The unique nature of 
nanocrystals solicits fabrication of new materials of controlled features [9]. The likely development of nanoparticles technology is an obvious long-term benefit [10]. With the success of assembling colloidal matter in a useful structure, the atoms and molecules will be treated as materials [11]. The investigation of the dynamics of an individual nanoparticle should be taken as a prime concern prior to go for further solid deliberations [12]. A good understanding on the surface features of nanoparticles will lead the development of higher order materials [13]. Tiny-sized clusters possess molecular-like electronic structures and non-fcc geometric structures [14]. Chemical properties of gold nanoparticles change with size [15].

It has been suggested that localized dynamics of the process is one of the cause that helps to evolve the structure of tiny particles in gold [16-20], silver [20], and carbon [21, 22]. Under the process of synergy or impinging electron streams from the regular external source, electron states of atoms are stretched as per available room while they remained bonded in the tiny particles [16]. A tiny-sized particle where it doesn't have certain shape, it goes for packing under mixed behavior of the field force [17-20]. The atoms of tiny particles reveal different modes of stretching electron states depending on the process of synergy or external source of impinging electron streams [16]. However, where impinging of electron streams is regular from the connected external source, the underlying atoms of tiny particles show orientational-based stretching of electron states [16]. The stretching of electron states of atoms while occupied tiny particles can be orientational-based under the process of synergy too [16]. When the stretching of electron states of an atom is orientational based, it is said to be the elongation of atom and when it is non-orientational based, it is said to be the deformation of atom and all those atoms of solid state behavior with at least one unfilled electron state reveal such behaviors as long as dealing various sorts of interactions to their counterparts [16-24].

Sir Isaac Newton explained the gravity called Newtonian Physics. There is no such concept opposite to gravity in the Theory of General Relativity as explained by Sir Albert Einstein. Bohr proposed that electrons move around the nucleus in allocated orbits where they have fixed energy as long as atom is in ground state. Therefore, the levitation behavior existing with full clarity at electronic level in atoms of various nature (but other than inert gas and hydrogen) remains beyond the understandings and the 
concept of band gap, valence band and conduction band dominated through all along with shells and orbital configurations, thus, was kept away to consider and think on 'force energy' directed phenomena safeguarding the smallest entity of matter known as electron in an atom in the form of filled state and where not safeguarding the smallest entity, it is called unfilled electron state which is always at bare surface of the atom. The more efforts were remained in exploring technological advances and breakthroughs and the efforts were put forth toward basic and fundamental sciences along with continuity in the explored ones remained in less focus.

In this work, the phenomena of heat and photon energy have been discussed and then how the electron at target in silicon atom utilized certain amount of heat energy followed by configuring scattered energy (of tits and bits) under the trajectory into photon energy -a propagating or travelling energy in discrete manner. Here, charisma of an atom of solid state behavior executing electronic transition under confined electron-dynamics is discussed where elastically-driven electronic state behavior configured force energy wavelength within inter-state electron's gap. The analogy between electron and photon is explained by taking silicon atom as a model system.

\section{RESULTS AND DISCUSSION:}

Under the application of suitable photonic current where wavelength of photons is in inter-state electron's gap, their propagation through inert gas atoms resulted into split them into electron streams [24]. Those electron streams are being utilized to impinge underlying atoms of tiny-sized particle resulting into transfer their backing force energy to deform them or elongate them depending on the mechanism of stretching electron states of atoms as discussed in the case of an atom embedded in tiny particle of twodimensional structure [24]. In either case where impinging of electron streams to underlying atoms of monolayer tiny particle is not for fixed period or the structure of tiny particle is not in two-dimension, the stretching of electron states is non-orientational based, which is termed as atomic deformation. Therefore, electronic structures of such behavior where atoms deform instead of elongating don't qualify to modify into smooth elements in the course of dealing travelling photons of adequate energy (wavelength in the range of hard X-rays) at their surfaces. A gold tiny shaped particle was taken as a 
model system to explain the mechanism of elongation along with modification into smooth elements where that reveal the localized gravity at solution surface due to certain geometric feature [23]. Such modification of atoms while they remained embedded into tiny-sized particle deal diffusion mechanism (stretching or migration) of electron states in certain orientation depending on the mechanism of impinging electron streams and this results into convert to smooth elements on dealing traveling photons of adequate wavelength by their electronic structure. The Fick's laws explain the mechanisms of diffusion of atoms and grains (mass flow) in various materials with respect to concentration both in linear and space steady state behaviors. Our previous study revealed diffusion mechanism of atoms and tiny particles where origin of diffusion was stretching of electron states [19].

Impinging electrons from the external source, in fact, utilize backing force energy as the heat to underlying atoms, on connection, which is being transferred to them in the form of kinetic energy to either deform or elongate under thermal behavior. Heat energy penetrate/enter to the medium directly as well via immersed/connected utilities known as cathode and anode in the form of propagating photonic current. The heat energy is resulted when a photon of certain length even a 'unit photon' merges or squeezes under the coordination or interaction of the medium. A unit photon has shape-like Gaussian distribution as shown in Figure 1 of (1). On squeezing or merging, a unit photon transforms force energy into heat energy. In the course of merging, a unit photon compresses the arm resulting into build unit heat energy called shunt energy as shown in Figure 1 of (2). In the course of squeezing, a unit photon compresses the arm while squeezing resulting into build unit heat energy called shunt energy as well, which is shown Figure 1 of (3). However, when a merged photon or a squeezed photon further divides into tits and bits, they purely determine the heat where the element of force is diminished, thus, dissipated the energy to medium uniformly, as in water, thus, tits and bits of heat energy are not called shunt energy. Distribution of heat to the medium is as per first law of thermodynamics. Such tits and bits of heat energy known as phonons have several options of splitting/dividing in either case, resulted on merging of unit (or overt) photon or on squeezing of unit (or overt) photon, as shown in Figure 1 of (4) and (5), respectively. Tits and bits of heat are placed around the atoms, thus, atoms like 
silicon element accumulate under the trajectory of their electrons while executing confined dynamics (inter-state dynamics) resulting into configure them in force energy.

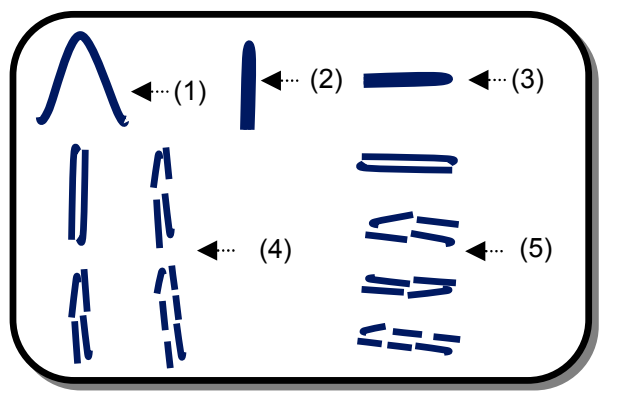

Figure 1: (1) The smallest entity of force energy shape-like Gaussian distribution called unit photon, on merging it is called (2) merged photon and on squeezing it is called (3) squeezed photon where (4) merged photon and (5) squeezed photon transformed into tits and bits of heat energy.

On entering photons of greater wavelengths, they raise the temperature of medium (solution/water) to greater volume but under less degree of hotness and vice versa. Similarly, long length photon which termed as overt photon carries more energy as a whole unit compared to the one called unit photon or shorter length photon, thus, raises the temperature of medium for less time at particular instant. As the heat entities deal localized energy as per nature of photons remained at background, thus, they can't deal the force when merged or squeezed under the interaction or coordination of specific nature medium; the element of force diminished because of the merging or squeezing photons completely or under their mixed behavior. Such heat entities travel (or propagate) through the medium like water, solutions of different composition, or other suitable mediums not working as an insulator, thus, obey the first law of thermodynamics. The word propagate is viable when photons are not completely converted into heat entities (tits and bits) transferring into inter-state electron's gap of water species and associated containments (components) where in addition to conduct heat medium (water pulse containments) also start conduct photonic current. In this context, sometime a living body deals shock known as electric shock on touching wire to water.

A photon characteristic current, in any length, propagates in inter-state electron's gap as the passage is feasible to propagate force energy of that photon. However, we can observe the burst or damage of copper or steel wire in the case of halting propagation 
of photons characteristic current either due to distributed inter-state electron's gap or their exceeded density propagation. The length of photon depends on the number of cycles counting at the point of generating source until the interruption takes place. As long as the dynamics of electron (at work) remains operational and consistent while retaining motion in inter-state (filled to unfilled state and unfilled to filled state at bare surface of the atom) it remains uninterrupted where configuring force energy under the connecting cycles of electron's trajectory is like a wave as shown in Figure 2 of (1); it is also called an overt photon when the electron configuring force energy comes at rest to fill its original state. Merged photons or squeezed photons are the subset of overt photon in a manner that they are resulted on coordinating or interacting overt photon to the suitable medium while placing horizontally or perpendicularly to certain nature interface. Under appropriate coinciding to a medium, an overt photon is transformed into merged photons (2) or squeezed photons (3) depending on the nature of interaction/coordination. However, when interaction or coordination of an overt photon remains normal to the surface of an interface, it is directly converted into squeezed photons; thus, they may not require further compression to become the recipe of heat. An overt photon contained the force energy of at least two unit photons; thus, it involves at least two nodes and two antinodes.

Due to interaction/coordination of travelling photons to medium, overt photons transformed into merged photons or squeezed photons and followed further division into tits and bits as well depending on the process. Now, on coordinating medium, an overt photon is transformed into merged photons, while on interacting medium, an overt photon transformed into squeezed photons and vice versa. As overt photon is the multiple of unit photon, thus, it merges into 'merged photons' while placing (travelling) parallel (horizontally) to the medium as shown in Figure 2 of (2), whereas, it squeezes into 'squeezed photons' while placing (travelling) perpendicular (vertically) to the medium as shown in Figure 2 of (3). Thus, the difference in merged photon and squeezed photon is the different process of collapsing of their parent photon, which is called overt photon. Heat energy of squeezed/merged photons can be greater than unit photon but still in discrete manner as encircled in Figure 2 and this may exactly match 
as well to free the electron at target from the inherent force of atom having different nature.

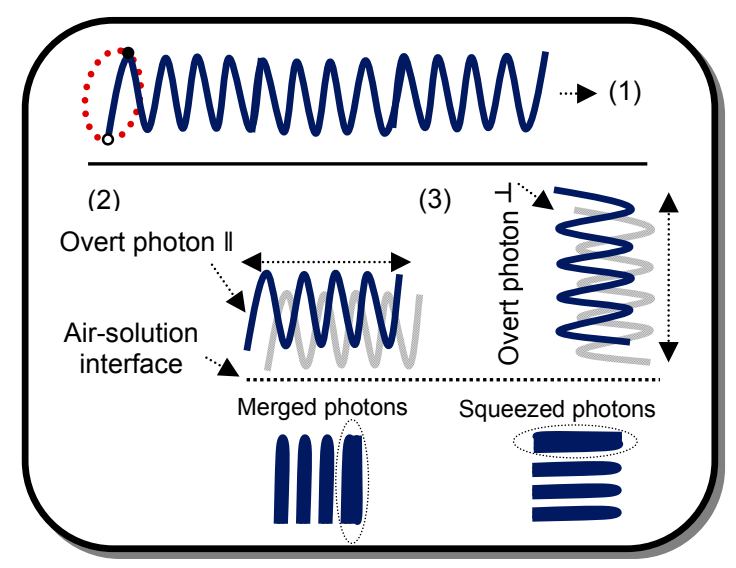

Figure 2: (1) Force energy configured under the trajectory of an electron while confined dynamics shows propagation in a wave-like fashion in inter-state electron's gap, (2) an overt photon on placing horizontally (II) at air-solution interface and (3) an overt photon on placing vertically $\left({ }^{\perp}\right)$ at air-solution interface resulted into merged photons and squeezed photons, respectively.

It infers that a merged photon or a squeezed photon owns discrete energy but doesn't the one where energy was configured in Gaussian distribution like shape, therefore, they deal energy related to heat which may be the amount of energy required to free an electron (at target) from the inherent force of its atom to go into excited state where levitation behavior prevailing at electronic level is working, however, that electron restores position under the force (gravitational) of nearby vacant state available at bare surface to keep the energy of that atom conserved. The heat energy at shunt level may vary atom to atom depending on the mass of an electron along with the intrinsic nature of the element. However, heat energy resulted on collapsing of unit photon, either by merging process or by squeezing process, is remained the same to free an electron to inherent force of nucleus in an atom from which that unit photon generated as in the case of silicon atom where heat energy resulted on collapsing of own unit photon rightly becomes suitable to free electron at target from the inherent force. Thus, different mass and amount of electrons in an atom embedded through the wrapped force energy result into varying the temperature of materials at atomic scale, nano scale, micro scale and bulk scale. 
The energy of merged photons (or squeezed photons) is related to heat energy, which is being transferred to suitable atoms under certain arrangement. Such heat energy can't enable electronic excitation in the inert gas atoms due to all filled states and room available to keep the energy conserved is already full, thus, they don't react and they don't reveal affinity with competence to atoms of other elements even with the atoms of their own family. However, atoms of suitable electronic transitions absorb (intake) such heat energy resulting into excite electron at target. Entering of heat energy (at shunt level) results into free electron from inherent force of its atom. 'Shunt energy' forces the electron from the back side available at the bare surface, frees it from the inherent force of the reimaging part of the atom, which results into excite that electron resulting into configure energy under the trajectory in the integral symbol, when electron comes back under the gravitational force of nearby available unfilled state at bare surface resulting into configure energy shape-like opposite to integral symbol in de-excitation period. Thus, the overall energy configured under the trajectory of electron has the shape-like Gaussian distribution and is the force energy called unit photon.

When heat energy of merged photons (or squeezed photons) coordinates to atom like silicon, providing shunt energy to its electron, it is being excited to deal the levity. As observed in solar panel at $45^{\circ}$ angle (approx.) with respect to plane oriented to the south will result into an average maximum power generated throughout the year where silicon atoms in the lattice not only absorbed the heat energy of made merged photons or squeezed photons but also dealt the direct absorption of such photons resulting into bonus power. Therefore, solar panels based on silicon atoms, the probability of exciting electrons becomes higher and systematic resulting results into long length photons, thus, an effective generation of current. Electron-dynamics are restricted within interstate electron's gap as discussed elsewhere [25]. The cycles of excitation and deexcitation of electrons in silicon atoms are non-stop for longer period where on titling silicon solar panel at certain orientation with respect to base results into varying the efficiency which is quite effective in the peak hours of sunlight.

In excitation of an electron from the filled state available at bare surface of its atom, the energy configured under the trajectory of electron was mainly due to levitation behavior when electron was de-excited under its free fall, the energy configured under 
the trajectory of electron was mainly due to gravitation behavior. That electron included the behaviours of inertia at the start, in between the curve of steady-state levitation and gravitation behaviours, and at the end as well. Thus, overall, configured energy is in the shape-like Gaussian distribution, which is the force energy called unit photon. This is a complete cycle of the electron being released from the inherent force of its atom while absorbing heat energy equal to shunt level. The same amount of shunt energy is being involved to free that electron again for re-exciting. Continuous phenomenon of excitation and de-excitation of electron within inter-state electron's gap results into an unterminated wave wavelength equal to inter-state electron's gap. As in Figure 3 where excitation of the electron (black color) under shunt energy is shown.

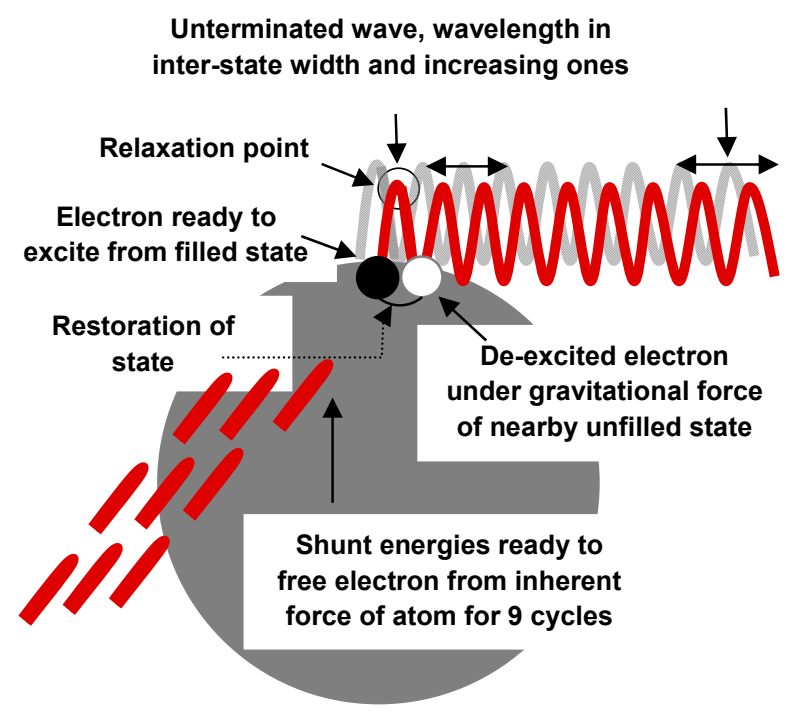

Figure 3: Electron at target free from the inherent force of atom is under shunt energy, excitation of electron is under levity and de-excitation of electron under gravity of nearby unfilled state at bare surface where repeated inter-state electron cycles results into energy in a wave-like fashion, which is increasing wavelength with respect to point of generation while entering shunt energy to re-excite for 9 cycles.

The energy configured along the trajectory where electron changed the state under its confined inter-state dynamics was due to inertia while the energy configured under the trajectory in steady-state behaviors of electron was due to levitation and gravitation behaviors. The process of self-returning of same electron to original state after completing 9 cycles shaped the energy like a wave as shown in Figure 3 and the interruption at the point of source generating it, it becomes the overt photon. The same process takes place in many atoms of the lattice which results in generating photonic 
current under some additional modification required for a silicon solar cell. The heat energy of merged photons or squeezed photons at shunt level required to excite the same electron at target 9 times is shown in Figure 3. De-excited electron will arrive at nearby vacant state available at bare surface as well due to dealing force of gravity, each time, and due to topology of an atom, thus, configuring energy shape-like Gaussian distribution against each cycle. However, continued supply of shunt energy to that electron in each cycle of de-excitation to re-excite will lengthen the wave. The force of repulsion is due to levitism phenomenon while force of attraction is due to gravitism phenomenon. Thus, in various so-called magnetic materials, the force of repulsion and attraction is not due to magnetism but relates to levitism phenomenon and gravitism phenomenon, respectively, where inertia is exempted due to available electrons at bare surface and intrinsic nature of atoms of those materials, for example, in iron, nickel and cobalt a pronounced behavior of levitism/gravitism is observed while dealing field force behavior in either way.

Therefore, the shunt energy enters at the backside of the electron available first at bare surface of the atom resulting into free it from the inherent force, thus, it goes to excited state where energy is configured under made trajectory due to inertia following by levity and then again due to inertia. The overall shape of configured energy is like integral symbol $\left(\int\right)$. While in the excited state and on reaching at certain point electron intends to relax as per intrinsic nature of its atom as shown in Figure 3 but changes state of rest to state of motion where energy is configured under made trajectory due to inertia following by gravity and then again due to inertia where overall shape of the configured energy is in opposite shape of integral symbol. The tensing of excited electron at relaxation point and filling nearby unfilled state is due to gravitational force acting at point of cavity size in the mass of the electron. So, the energy configured in the shape of two integration symbols, opposite to each other, remained connected under the path of electron resulted into give the overall shape of configured energy like Gaussian distribution as shown in Figure 4. 


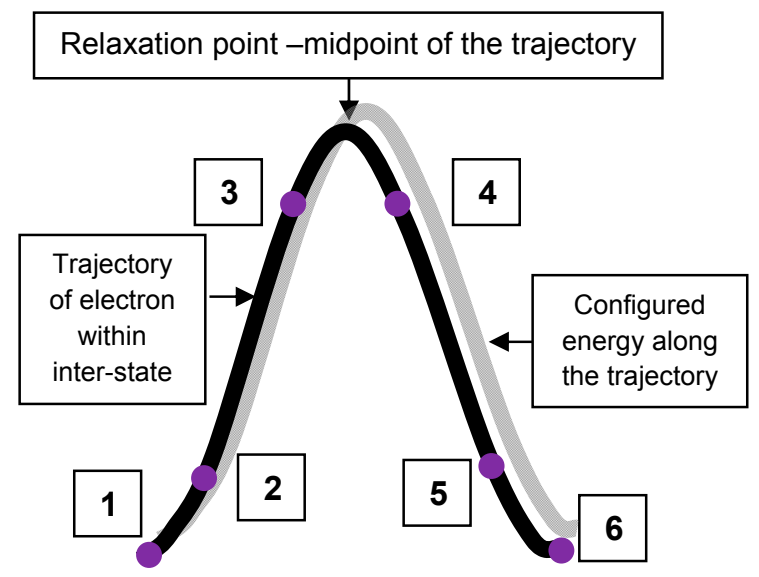

Figure 4: An electron showing configured energy shape-like Gaussian distribution under trajectory front side (dark color) shows trajectory followed by the electron while exciting from original filled state to relaxation point following to vacant state and rear side (grey color) shows configured force energy under (along) the trajectory of electron.

The energy configured along the path when an electron is going to excite under the disturbance of its state of rest, it is due to inertia of the electron, as it is reaching in the state of motion to excite upto relaxation point - (1) to (2) in Figure 4. In the time of exciting the electron, the energy configured under the trajectory is due to steady-state levitation behavior - between (2) and (3) in Figure 4. On turning the electron to go into relaxation, the energy configured is due to inertia of the electron - (3) to mid-position of the curve in Figure 4. When the electron is turned into de-excitation, instead of relaxing at relaxation point, energy configures due to inertia of the electron - mid-position of the curve to (4) in Figure 4. In the course of de-exciting the electron, the energy configured under its trajectory is due to steady-state gravitation behavior - between (4) and (5) in Figure 4. The energy configured on reaching to ground position of electron is again under the inertia as it changed the state from motion to rest - (5) to (6) in Figure 4. An equal amount of inertia of electron involved since, to start motion of electron from rest or come at rest from its motion. Thus, without any breakings, those portions of energy configured under confined electron-dynamics giving out the force energy which is termed as 'unit photon'.

In this context, at one side heat energy is dealing matter at atomic to enabling free an electron from the inherent and at other side scattered heat energy in tits and bits configuring along the trajectory, thus, converting into force energy, which is related to 
photon energy. That photon energy works again as the heat energy while interacting/coordinating to suitable medium, thus, working again as the input energy of dedicated atoms where transforming energy related to heat into energy related photon again -a force energy. In this context, heat energy dedicates suitable atoms to configure it into photon energy. Thus, a photon is the 'force energy', which propagates in inter-state electron's gap, thus, envisage useful and controlled usage. On the other hand, when travelling such photons, more often, in normal air medium where lose force by dissipating energy called heat under continuous increase of their wavelength. The origin of photon energy is the energy resulted due to heat and configuring under the confined electron-dynamics. In the normal air environment, force energy of the travelling photon is being forced by the surrounding environment, thus, dissipated the energy in the accompanying environment in the form of heat, but resulting into increase the wavelength of travelling photon but decreasing the force energy against that.

As discussed elsewhere [21], the enhanced field emission characteristic of 'tiny grains carbon films' was related to modified graphitic phase tiny grains [21]; tiny grains modified electronic structure into smooth elements become the source of enhanced field emission and display panel applications. As photonic current propagates in inter-state electron's gap [24], hence, smooth elements of modified tiny grains deliver enhanced field, thus, their more formation in carbon thin films allow straight-forwardly utilization for field emission applications or display panels. The performance of such 'tiny grains carbon films' should be notified in terms of formation of maximum smooth elements of tiny grains followed by the nature of their structural connections and such films can give phenomenal controlled features as discussed elsewhere [21]. The same approach should be considered in studying phenomena like surface enhanced Raman scattering or localized surface Raman spectroscopy of nanoparticles [26]. Because those phenomena are large studied in the metallic materials where nanoparticles/particles of anisotropic shapes deliver enhanced field due to made smooth elements of twodimensional structure as discussed in several studies, elsewhere [17-20].

Distribution of heat energy to disordered structure is in non-uniform manner. More heat is resulted in structures where atoms are randomly distributed as photons characteristic current merged or squeezed instead of their entire propagation via inter- 
state electron's gap of atoms embedded in the lattice. Collapsed photons in tits and bits can again transform into merged photons or squeezed photons, thus, working as the heat energy at shunt level to excite an electron of suitable atom. Similarly, when photons of different energy disrupt the medium (overt photons), they dissipate heat energy in the form of merged photons or squeezed photons. Photons assign different roles to matter on their interaction or coordination transforming into one form of energy to another depending on the structural motifs and individual nature of constituted atoms. In Bragg's diffraction, amorphous materials don't reveal any specific structure under the interaction of photons wavelength in X-rays where energy of photons mainly transformed into heat rather than visualized in the XRD scan. In some cases, photons characteristic current are utilized to split the matter like inert gas atoms and their electrons streams are being utilized to deform or elongate underlying atoms of electronic transitions [24]. In the stone's age, catching fire was discovered while striking an embedded stone to another stone under intensive acceleration where captured fluke energy at their point of contact converted into 'force energy', thus, under exceeding the wavelength in the air medium, on reaching in the visible range, glow of light appeared but prior to that reduction, the force energy under transformation into heat dissipated in the surrounding environment.

The set modalities of all sorts of photons depend on the origin of their generation establishing roles set by the manufacturer or expert under the coordination or interaction to suitable matter. In this context, structural design is crucial in targeting their specific application and many studies are now targeting and exploring structure either standalone or in relation to other fields of science [27-36]. Our several investigations discuss that structural motifs are owing to characteristics photons and field force behaviors [16-24] where it is proven experimentally in one of the study given elsewhere [18]. In addition to evolution of structure in graphitic phase, amorphous and modified phase of tiny grains carbon film also observed in Raman spectroscopy and energy loss spectroscopy [21]. The field force behaviours are critical depending on the filled/unfilled electron state (s) of atoms available at their bare surface, thus, dealing some atoms in gaseous state, some in solid state but carbon work on both sides [37]. A recent study expressed the silent feature and implication of modified electronic structure while using 
as nanomedicine [38]. Our recent study shows how gravity prevailing at unfilled region of developing geometric anisotropic shape deal packing of equilateral triangular-shaped tiny particles [39].

On interrupting a wave generating at point of source under confined electrondynamics executing by a suitable atom, it resulted into long length photon called overt photon, on coordination or interaction to appropriate medium, either placing parallel to or perpendicular to, transforms into merged photons or squeezed photons, respectively, following by transformation into heat energy and where a photon called 'unit photon' merged or squeezed, it results into heat energy called shunt energy. Electrons excite in atoms of certain nature under that heat energy called shunt energy on entering at their backside. Whereas, those atoms don't execute electronic transitions, split under the application of photonic current following by increasing wavelength as discussed elsewhere [24]. Therefore, shunt energy is a quantized energy which is required to free an electron from the inherent force of remaining part of atom, thus, that is a multiplied of unit photon heat energy when dealing electron of multiplied mass. To measure temperature of such materials and in their selective sizes is the integral part to understand science of their different behaviours and some of the studies shed light on it [40-42]. It is possible to measure temperature of atoms, their nanoscale components and at bulk scale as well without the involvement of thermocouples and introducing various gadgets, theories, etc. What is required, to determine the probability of exciting electrons at shunt energy in the atoms of solid state behavior execute electronic transitions? In the greater mass of electron that heat energy multiplied resulting into give shunt energy of releasing a heavier electron from the force of its remaining part of atom.

An electron has mass and it is diffused under the deformation or stretching process of certain nature atoms. In the case of photon, it doesn't have mass and it involves only discrete energy, which is being forced. In this context, overt photons neither diffused nor impinged and they even don't strike, they either propagate or travel where their energy is being absorbed by the coordinated (or interacted) medium. The nature of coordinated photon itself signified as the energy reveals different behavior; propagate as well as travel. Photons wavelength in visible range enable resolution of the image down to 0.2 
$\mathrm{mm}$. Photons energy in the wavelength of X-rays propagate through human body and identify the sign of fracture. Photons characteristic current propagate in suitable wire, thus, they are photonic current. Different cameras and devices manipulate the image on screening reflected photons at the surface of an object. When it is said that an electron is spoiled it doesn't mean that the atom of that electron is ionized but it means that the electron occupying atom is no more under elastically-driven behavior and atom enters in the zone where electron is dealing plastically-driven behavior, thus, such atoms either deform or elongate but do not ionize [24]. A recent study explained the role of van der Waals interactions in the case of isolated atom by considering the induced dipoles [43], which can be attained when fluctuations of charge density are in wave-like nature [44].

\section{CONCLUSIONS:}

When a merged or a squeezed photon squeezing arms under the inward compression, it directly provides heat energy to electron of the atom at target as long as it doesn't divide into tits and bits. Overt photons are long length photons where an overt photon is transformed into merged or squeezed photons under the coordination or interaction of suitable medium. That heat energy at shunt level enables an electron to free from the inherent force of inner part of atom known as nucleus. Those photons transformed into tits and bits of heat utilized to configure again into photon under inter-state confined trajectory of electron where force energy depends on the number of uninterrupted confined electron's cycles. A photon resulted under single cycle inter-state electron's excitation de-excitation where inertia was involved is called a unit photon. Exciting electrons under confined dynamics while supplying uninterrupted heat energy results into immeasurable length of energy travelling in a wave-like fashion, on interruption called a photon and on collapsing called heat energy. On interrupting the energy configuring in a wave-like fashion at the point of source of generating, it becomes the overt photon, which is a long length photon, when coordinated or interacted to suitable medium, it transforms into either merged photons (on parallel placing to medium) or squeezed photons (on perpendicular placing to medium). Thus, heat energy and photon energy work one after the other where intermediate component is a suitable matter. On collapsing photons by the suitable medium resulted into heat energy and on 
reconfiguring that heat energy via suitable atoms again transformed into photon energy. Therefore, those atoms are naturally built-in machines.

In various materials, structural adjustments to different forms of energy find the way out for certain application, indicating that design of the material manipulates energy for targeted application. Photons characteristic current, when it collectively propagates in suitable wire, it is a photonic current. An electron has strong analogy to photon in a manner that inter-states electron's motion result into configure energy shape like a wave where at each state of changed motion, inertia is involved.

Structure of certain materials deliver straight-forward application on interacting or coordinating (or both) to heat energy or photon energy, for example, solutions of various metallic colloids and quantum dots, on interacting light, split light into different colors depending on the refraction reflection modes along with size and shape of the nanoparticles/particles and 'tiny grains carbon films' give enhance field emission characteristic on the basis of aligned inter-state electron's gap in carbon atoms dealing graphitic phase. When inertia is involved depending on the nature of electron states of an atom, the resulted behavior of electron deals it as well, whenever changed the state and responsible for propagating/travelling the energy in a wave-like fashion. All those atoms of solid state behavior execute electronic transitions are naturally built-in machines (devices) to utilize heat energy and transform it into photon energy and atoms of silicon lattice are the model system.

The heat energy utilized to free an electron from the force of remaining part of atom is being utilized to release it only and it has nothing to do with energy configuring under electron's trajectory. The energy placed under the trajectory of electron from the surrounding medium while in exciting period deal inertia-levity-inertia behaviours where energy configured in integral symbol. However, while in de-exciting period deal inertialevity-inertia behaviours where energy configured in inverted integral symbol. An electron is a matter, perhaps, the smallest entity of matter and it is the most valuable when it works within natural confinement.

Clearly, electrons are matter, occupy space, possess mass and impinge (or strike) under taken energy from external source while they excite to relaxation point from bare surface of their atoms under the heat energy at shunt level and de-excite under the 
cavity of unfilled states available at the bare surface of their atoms as well. Whereas, all types of photons possess energy as well as force but not mass, they use space in propagation within the gap of their source of generation (inter-state electron's gap) where their wavelength remain confined. However, while their travelling in normal air medium, they dissipate their force energy into heat, thus, decreasing the wavelength, so, they are not the ones impinging (or striking) or exciting (de-exciting) under confined dynamics.

\section{References}

[1] D. Bohm, D. Pines, A Collective Description of Electron Interactions. I. Magnetic Interactions, Phys. Rev. 1951, 82, 625-634.

[2] D. Pines, D. Bohm, A Collective Description of Electron Interactions: II. Collective vs Individual Particle Aspects of the Interactions, Phys. Rev. 1952, 85, 338-353.

[3] R. H. Ritchie, Plasma Losses by Fast Electrons in Thin Films, Phys. Rev. 1957, 106, 874-881.

[4] D. Bohm, D. Pines, A Collective Description of Electron Interactions: III. Coulomb Interactions in a Degenerate Electron Gas, Phys. Rev. 1957, 92, 609-625.

[5] T. Low, et al., Polaritons in layered two-dimensional materials, Nat. Mater. 2017, 16, 182-194.

[6] J. Frenkel, On the Transformation of Light into Heat in Solid. I, Phys. Rev. 1931, $37,17-44$.

[7] O. D. D. Couto Jr. et al., Charge control in $\operatorname{lnP} /(\mathrm{Ga}, \mathrm{In}) \mathrm{P}$ single quantum dots embedded in Schottky diodes, Phys. Rev. B 2011, 84, 125301-7.

[8] M. Brust, M. Walker, D. Bethell, D. J. Schiffrin, R. Whyman, Synthesis of Thiolderivatised Gold Nanoparticles in a Two-phase Liquid-Liquid System, J. Chem. Soc., Chem. Commun. 1994, 801-802.

[9] R. L. Whetten, J. T. Khoury, M. M. Alvarez, S. Murthy, I. Vezmar, Z. L. Wang, P. W. Stephens, C. L. Cleveland, W. D. Luedtke, U. Landman, Nanocrystal Gold Molecules, Adv. Mater. 1996, 8, 428-433.

[10] M. Brust, C. J. Kiely, Some recent advances in nanostructure preparation from gold and silver particles: a short topical review, Colloids and Surfaces A: Physicochem. 
Eng. Aspects 2002, 202, 175-186.

[11] S. C. Glotzer, M. J. Solomon, Anisotropy of building blocks and their assembly into complex structures, Nature Mater. 2007, 6, 557-562.

[12] S. Link, M. A. El-Sayed, Shape and size dependence of radiative, nonradiative and photothermal properties of gold nanocrystals, Int. Rev. Phys. Chem. 2000, 19, 409453.

[13] C. P. Shaw, D. G. Fernig, R. Lévy, Gold nanoparticles as advanced building blocks for nanoscale self-assembled systems, J. Mater. Chem. 2011, 21, 12181-12187.

[14] Y. Negishi, T. Nakazaki, S. Malola, S. Takano, Y. Niihori, W. Kurashige, S. Yamazoe, T. Tsukuda, H. Häkkinen, A Critical Size for Emergence of Nonbulk Electronic and Geometric Structures in Dodecanethiolate-Protected Au Clusters, J. Am. Chem. Soc. 2015, 137, 1206-1212.

[15] A. Moscatelli. Gold nanoparticles: Metallic up to a point, Nature Nanotechnol. 2015, DOI:10.1038/nnano.2015.16.

[16] M. Ali, I -Nan. Lin, The effect of the Electronic Structure, Phase Transition and Localized Dynamics of Atoms in the formation of Tiny Particles of Gold. arXiv:1604.07144v5.

[17] M. Ali, I -N. Lin, Development of gold particles at varying precursor concentration. arXiv:1604.07508v6.

[18] M. Ali, I -N. Lin, Controlling morphology-structure of particles at different pulse rate, polarity and effect of photons on structure. arXiv:1605.04408v6.

[19] M. Ali, I -N. Lin, Formation of tiny particles and their extended shapes-origin of physics and chemistry of materials. arXiv:1605.09123v7.

[20] M. Ali, I -N. Lin, Tapping opportunity of tiny shaped particles and role of precursor in developing shaped particles. arXiv:1605.02296v6.

[21] M. Ali, I -Nan. Lin, Phase transitions and critical phenomena of tiny grains thin films synthesized in microwave plasma chemical vapor deposition and origin of $\mathrm{v} 1$ peak. arXiv:1604.07152v7.

[22] M. Ali, M. Ürgen, Switching dynamics of morphology-structure in chemically deposited carbon films-a new insight, Carbon 2017, 122, 653-663. 
[23] M. Ali, The study of tiny shaped particle dealing localized gravity at solution surface. arXiv:1609.08047v6.

[24] M. Ali, Atoms of electronic transition deform or elongate but do not ionize while inert gas atoms split. arXiv:1611.05392v7.

[25] M. Ali, Structure evolution in atoms of solid state dealing electron transitions. arXiv:1611.01255v6.

[26] S. Nie, S. R. Emory, Probing Single Molecules and Single Nanoparticles by Surface-Enhanced Raman Scattering, Science 1997, 275, 1102-1106.

[27] J. Zhao, L. Yang, Structure Evolutions and Metallic Transitions in $\ln _{2} \mathrm{Se}_{3}$ Under High Pressure, J. Phys. Chem. C 2014, 118, 5445-5452.

[28] V. N. Manoharan, Colloidal matter: Packing, geometry, and entropy, Science 2015, 349, 1253751.

[29] J. Park, et al., 3D structure of individual nanocrystals in solution by electron microscopy, Science 2015, 349, 290-295.

[30] D. Jacobsson, et al., Interface dynamics and crystal phase switching in GaAs nanowires. Nature 2016, 531, 317-322.

[31] T. Tuma, A. Pantazi, M. L. Gallo, E. Eleftheriou, Stochastic phase-change neurons. Nature Nanotech. 2016, 11, 693-699.

[32] J. Rensberg, et al., Active Optical Metasurfaces Based on Defect-Engineered Phase-Transition Materials, Nano Lett. 2016, 16, 1050-1055.

[33] Y. Suzuki, G. Cardone, D. Restrepo, P. D. Zavatteri, T. S. Baker, F. A. Tezcan, Selfassembly of coherently dynamic, auxetic, two-dimensional protein crystals, Nature 2016, 533, 369-373.

[34] C. H. J. Evers, J. A. Luiken, P. G. Bolhuis, W. K. Kegel, Self-assembly of microcapsules via colloidal bond hybridization and anisotropy, Nature 2016, 534, 364368.

[35] I. R. Epstein, B. Xu, Reaction-diffusion processes at the nano- and microscales. Nature Nanotech. 2016, 11, 312-319.

[36] A. Azizi, Spontaneous Formation of Atomically Thin Stripes in Transition Metal Dichalcogenide Monolayers, Nano Lett. 2016, 16, 6982-6987. 
[37] M. Ali, Why some atoms are in gaseous state and some in solid state but carbon work on either side (2017). (Submitted for consideration).

[38] M. Ali, Nanoparticles-Photons: Effective or Defective Nanomedicine, J. Nanomed. Res. 2017, 5(6), 00139.

[39] M. Ali, I -N. Lin, C. -J. Yeh, Predictor packing in developing unprecedented shaped colloidal particles (submitted for consideration).

[40] M. Mecklenburg, W. A. Hubbard, E. R. White, R. Dhall, S. B. Cronin, S. Aloni, B. C. Regan, Nanoscale temperature mapping in operating microelectronic devices, Science 2015, 347, 629-632.

[41] L. Ye, D. Hou, X. Zheng, Y. Yan, M. D. Ventra, Local temperatures of stronglycorrelated quantum dots out of equilibrium, Phys. Rev. B 2015, 91, 205106-8.

[42] F. Menges, et al., Temperature mapping of operating nanoscale devices by scanning probe thermometry, Nat. Commun. 2016, 7:10874 doi: 10.1038/ncomms10874.

[43] S. Kawai, et al., Van der Waals interactions and the limits of isolated atom models at interfaces, Nat. Commun. (2016) DOI: 10.1038/ncomms11559.

[44] A. Ambrosetti, N. Ferri, R. A. DiStasio Jr., A. Tkatchenko, Wavelike charge density fluctuations and van der Waals interactions at the nanoscale, Science 2016, 351, 1171-1176.

\section{Author's biography:}

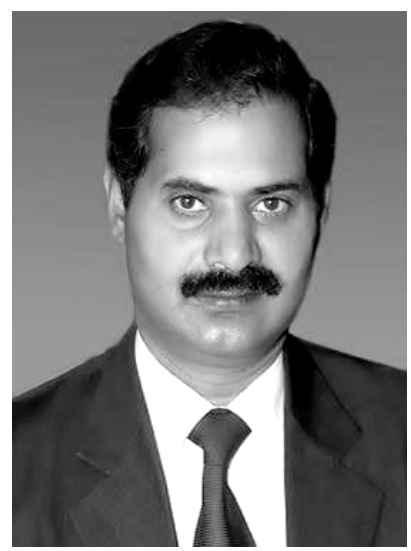

Mubarak Ali graduated from University of the Punjab with B.Sc. (Phys\& Maths) in 1996 and M.Sc. Materials Science with distinction at Bahauddin Zakariya University, Multan, Pakistan (1998); thesis work completed at Quaid-i-Azam University Islamabad. He gained Ph.D. in Mechanical Engineering from Universiti Teknologi Malaysia under the award of Malaysian Technical Cooperation Programme (MTCP;2004-07) and postdoc in advanced surface technologies at Istanbul Technical University under the foreign fellowship of The Scientific and Technological Research Council of Turkey (TÜBITAK; 2010). He completed another postdoc in the field of nanotechnology at Tamkang University Taipei (2013-2014) sponsored by National Science Council now M/o Science and Technology, Taiwan (R.O.C.). Presently, he is working as Assistant Professor on tenure track at COMSATS Institute of Information Technology, Islamabad campus, Pakistan (since May 2008) and prior to that worked as assistant director/deputy director at M/o Science \& Technology (Pakistan Council of Renewable Energy Technologies, Islamabad; 2000-2008). He was invited by Institute for Materials Research (IMR), Tohoku University, Japan to deliver scientific talk on growth of synthetic diamond without seeding treatment and synthesis of tantalum carbide. He gave several scientific talks in various countries. His core area of research includes materials science, physics \& nanotechnology. He was also offered the merit scholarship (for PhD study) by the Government of Pakistan but he couldn't avail. He is author of several articles published in various periodicals (https://scholar.google.com.pk/citations?hl=en\&user=UYjvhDwAAAAJ). 